\title{
Communications
}

\section{Catalytic Asymmetric Fluorination of $\alpha$-Chloro- $\beta$-ketoesters in the Presence of Chiral Palladium Complexes}

\author{
Min Je Cho, Young Ku Kang, Na Ri Lee, and Dae Young Kim" \\ Department of Chemistry, Soonchunhyang University, Asan, Chungnam 336-745, Korea. "E-mail: dyonng@sch.ackr \\ Received Angust 16, 2007
}

Key Words : Electrophilic fluorination, $\Lambda$ symmetric catalysis, Chiral palladium catalysts, $\alpha$-Clloro- $\beta$-keto esters, $\alpha$-Chloro- $\alpha$-fluoro- $\beta$-ketoesters

The chemistry of organic fluorine compounds is a rapidly developing area of research because of their importance in biochemical and medicinal applications and material science.' Introduction of fluorine atom into biologically active compounds often leads to improvement of chemical and physical properties of the parent compounds due to unique properties of the fluorine atom. ${ }^{2}$ Chiral organofluorine compounds containing a fluorine atom bonded directly to a stereogenic center have been utilized in studies of enzyme mechanisms and as intermediates in asymmetric syntheses. ${ }^{3}$ The development of effective methodologies for the preparation of stereoselectively fluorinated compounds having a fluorine atom at a stereogenic carbon center is critical in order to further advances of fluorine chemistry. ${ }^{4}$ Until now, a number of enantioselective fluorinations have been achieved by reagent-controlled and catalytic enantioseletive fluorination." Recently, several groups have been reported the catalytic enantioselective fluorination of active methine derivatives using chiral Lewis acids ${ }^{6}$ such as Binap-Pd(II) and transition metal-bis (oxazoline) complexes and organocatalysts ${ }^{7}$ such as cinchonine-derived quaternary ammonium salt, imidazolidinone, and proline derivatives.

A few synthetic methods for the preparation of $\alpha$-chloro$\alpha$-fluoro- $\beta$-ketoesters have been reported. In general, $\alpha$ chloro- $\alpha$-fluoro- $\beta$-ketoesters were prepared by the electrophilic fluorination of $\alpha$-chloro- $\beta$-ketoesters using NFSI or $\mathrm{F}_{2}$ in the presence of formic acid. ${ }^{8}$ Togni has recently reported

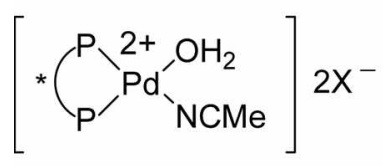<smiles>[R17]c1ccc2ccccc2c1-c1c(P)ccc2ccccc12</smiles>

3a: $\mathrm{Ar}=\mathrm{Ph}:(R)-\mathrm{BINAP}, \mathrm{X}=\mathrm{OTf}$

3b: $\mathrm{Ar}=\mathrm{Ph}:(R)-\mathrm{BINAP}_{1} \mathrm{X}=\mathrm{BF}_{4}$

3c: $\mathrm{Ar}=\mathrm{Ph}:(R)-\mathrm{BINAP}, \mathrm{X}=\mathrm{SbF}_{6}$

3d: $\mathrm{Ar}=\mathrm{Ph}:(R)$-BINAP, $\mathrm{X}=\mathrm{PF}_{6}$

3e: $\mathrm{Ar}=4$-methylphenyl: $(R)$-Tol-BINAP, $\mathrm{X}=\mathrm{PF}_{6}$

3f: $\mathrm{Ar}=3,5$-dimethylphenyl: $(R)$-Xylyl-BINAP, $\mathrm{X}=\mathrm{BF}_{4}$

Figure 1. Chiral palladiun complexes 3 . the first catalytic enantioselective synthesis of $\alpha$-chloro- $\alpha$ fluoro- $\beta$-ketoesters using chiral titanium complexes with up to $65 \%$ selectivity. ${ }^{9}$

As part of research program related to the development of synthetic methods for the enantioselective construction of stereogenic carbon centers. ${ }^{10}$ we reported the catalytic enantioselective $\alpha$-fluorination and $\alpha$-amination of $\beta$-ketoesters with excellent enantioselectivity which promoted by chiral ammonium salts and chiral palladium complexes. ${ }^{77.102}$ In this letter, we wish to report the catalytic enantioseletive electrophilic $\alpha$-fluorination of $\alpha$-chloro- $\beta$-ketoesters using chiral palladium complexes $\mathbf{3}$ which are air- and moisturestable.

To determine suitable reaction condition for the catalytic enantioselcetive fluorination of $\alpha$-chloro- $\beta$-ketoesters 1 , we first examined electrophilic fluorination of 1-chloro benzoylacetate 1a with $N$-fluorobenzenesulfonimide (NFSI) in the presence of $5 \mathrm{~mol} \%$ of 3a in $\mathrm{MeOH}$ at room temperature (Table 1). As can be seen from Table 1, the fluorinated

Table 1. Optinization of the reaction conditions

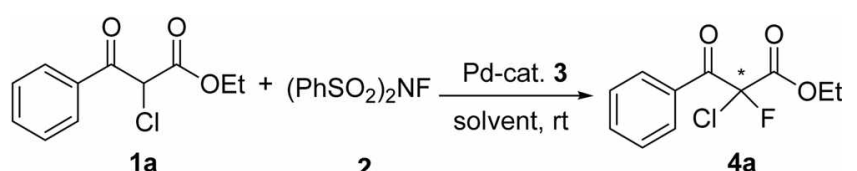

\begin{tabular}{cccccc}
\hline Entry & Catalyst & Solvent & Time (h) & Yield" (\%) & $\mathrm{Ee}^{\text {b }}(\%)$ \\
\hline 1 & 3a & $\mathrm{MeOH}$ & 118 & 87 & 65 \\
2 & 3b & $\mathrm{MeOH}$ & 66 & 65 & 61 \\
3 & 3c & $\mathrm{MeOH}$ & 118 & 88 & 65 \\
4 & 3d & $\mathrm{MeOH}$ & 72 & 90 & 75 \\
5 & 3e & $\mathrm{MeOH}$ & 72 & 71 & 67 \\
6 & 3f & $\mathrm{MeOH}$ & 72 & 71 & 66 \\
7 & 3d & Toluene & 72 & 41 & 69 \\
8 & 3d & Acetone & 72 & 88 & 67 \\
9 & 3d & THF & 72 & 74 & 67 \\
$10^{\circ}$ & 3d & MeOH & 125 & 85 & 11 \\
$11^{\prime \prime}$ & 3d & MeOH & 125 & 0 & - \\
\hline
\end{tabular}

"Tsolated yields. ${ }^{5}$ Enantiopurity was detemined by I IPLC analysis using with a chiralcel OB-I I column. "Reaction carried out using Selectfluor ${ }^{\text {Ty }}$ as fluorination reagent. "Reaction carried out using 1 -fluoropyridinium triflate as fluorination reagent. 
Table 2. Catalytic asymmetric fluorination of $\alpha$-chloro- $\beta$-ketoesters

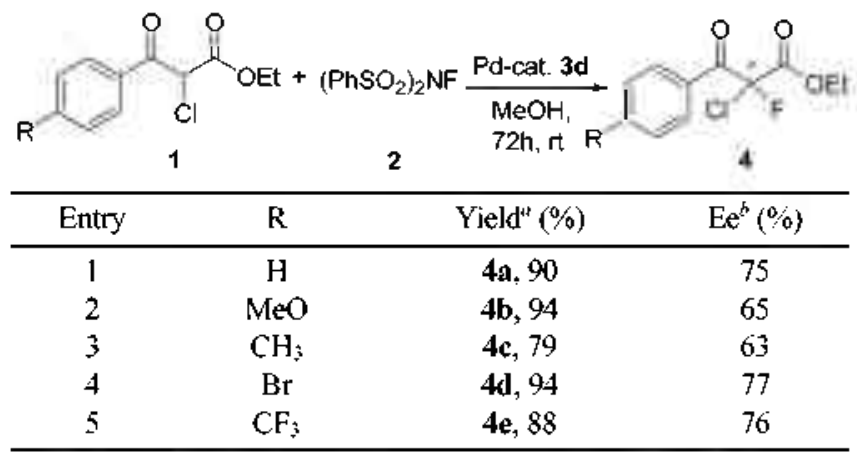

${ }^{a}$ Tsolated yields. "Enantiomeric excess deternined by chiral HPLC analysis using with a chiralcel $\mathrm{OB}-\mathrm{H}$ and $\mathrm{OJ}$ columns.

product was obtained with $87 \%$ yield with $65 \%$ ee after 118 h (entry 1). To improve the enantioselectivity, we examined a series of chiral diphosphine ligands. When $(R)$-phenylBINAP palladium complex $\mathbf{3 d}$ was used, the enantioselectivity was improved to $75 \%$ ee (entry 4 ). Conceming the solvent, there are no significant effects on enantioselectivity. NFSI was more effective fluorinating agent than Selectfluor in this reaction under the same condition (entries 4 and 10). Using 1-fluoropyridinium triflate as fluorination reagent under similar conditions, the reaction was not proceeded (entry 11).

To examine the generality of the catalytic enantioselective fluorination of $\alpha$-chloro- $\beta$-ketoesters 1 by using chiral palladium complex 3d, we studied the fluorination of $\alpha$ chloro- $\beta$-ketoester derivatives 1 under optimum reaction condition. As it can be seen by the results summarized in Table 2 , the corresponding $\alpha$-chloro- $\alpha$-fluoro- $\beta$-ketoesters 4 were obtained in moderate to excellent yields with high enantioselctivities (63-77\% ee). ${ }^{\prime \prime}$

In summary, we have accomplished the efficient catalytic enantioselective electrophilic $\alpha$-fluorination of various $\alpha$ chloro- $\beta$-ketoesters 1 with good enantioselectivity (up to $77 \%$ ee) using palladium complex $\mathbf{3 d}$ as chiral catalyst. It should be noted that this fluorination reaction proceeded well using air- and moisture-stable chiral palladium complexes in environmentally benign alcoholic solvent. This catalytic enantioselective fluorination in $\mathrm{MeOH}$ has been shown to be practical from environmental and economical points of view. Current efforts are toward developing synthetic applications of this a-fluorination reaction.

Acknowledgement. This research was supported by the Korea Research Foundation Grant by the Korean Government (KRF-2006-521-C00099).

\section{References}

1. For reviews: (a) Kirk, K. L. J. Fluorine Chem, 2006, 127, 10131029. (b) Isanbor, C.; O'Hagan, D. J. Fluorine Chem. 2006, 127, 303-319.

2. (a) Smart, B. E. J. Fluorine Chem. 2001, 109, 3-11. (b) Ismail, F. M. D. J. Fluorine Chem. 2002, 118, 27-33. (c) Bohm, H.-J.; Banmer, D.; Bendels, S.; Kansy, M.; Kuhn, B.; Muller, K.; ObstSander, U.; Stahl, M. ChemBioChem 2004, 5, 637-643.
3. Asymmetric Fhoroorganic Chemistiy: Sywthesis, Application, and Fiture Directions; Ramachandran, P. V., Ed.; ACS Symposium Series 746; American Chemical Society: Washington, DC, 2000.

4. For reviews: (a) Lal, G. S.; Pez, G P.; Syvret, R. G. Cheni. Rev. 1996, 96, 1737-1755. (b) Taylor, S. D.; Kotoris, C. C.; Hum, G. Tetrahedron 1999, 55, 12431-12477.

5. For reviews: (a) Prakash, G. K. S.; Bier, P. Angew. Chent. Int. Ed 2006, 45, 2172-2174. (b) Mikami, K.; Ioh, Y.; Yamanaka, M. Chem, Rev, 2004, J04, l-16. (c) Ibrahim, H.; Togni, A. Chem. Conmun. 2004. I147-1 155. (d) Ma, J.-A.; Cahard. D. Cheni. Rev: $2004,104,6119-6149$.

6. (a) Kang, Y. K.; Cho, M. J.; Kim, S. M.; Kim, D. Y. Sintent 2007. 1135-1138. (b) Kim, S. M.; Kang, Y. K.; Let, K.; Mang, J. Y.; Kim, D. Y. Bull. Korean Chem, Soc, 2006, 27, 423-425. (c) Kim, S. M.; Kim, H. R.; Kim, D. Y. Org. Lett. 2005. 7, 2309-231l. (d) Bernardi, L.; Jorgensen, K. A. Chem. Commin. 2005, 1324-1326. (e) Hamashima, Y.; Suzuki, T.; Shimura, Y.; Shimizu, T.; Umebayashi, N.; Tamura, T.; Sasamoto, N.; Sodeoka, M. Tetrahedron Lett. 2005, 46, 1447-1450. (f) Kim, H. R.; Kim, D. Y. Tetrahedron Lett. 2005. 46, 3115-3117. (g) Shibata. N.; Kohno, J.; Takai, K.; Ishimaru, T.; Nakamura, S.; Toru, T.; Kanemasa, S. Angew, Chem., Int, Ed, 2005, 44, 4204-4207, (h) Park, E. J.; Kim, H. R.; Joung. C. W.; Kim, D. Y. Bull. Korean Chem. Soc. 2004 , 25, 1451-1452. (i) Shibata, N.; Ishimanu, T.; Nagai, T.; Kohno, J.; Toru, T. Synlett 2004, 1703-1706. (j) Ma, J.-A.; Cahard, D. Tetrahedron: Asymmetry 2004, 15, 1007-1011.

7. (a) Huang, Y.; Walji, A. M.; Larsen, C. H.; MacMillan, D. W. C. J. An. Chem. Soc. 2005, 127, 15051-15053. (b) Enders, D.; Hutt1, M. R. M. Synlett 2005, 991-993. (c) Marigo, M.; Fielenbach, D.; Braunton, A.; Kjersgaard, A.; Jørgensen, K. A. Angew: Chem, Int. Ed. 2005, 44. 3703-3706. (d) Steiner, D. D.; Mase. N.; Barbas, C. F., III Angev. Chem, Int. Ed. 2005, 44, 3706-3710. (e) Beeson, T. D.: MacMillan, D. W. C. J. Anl. Cheml. Soc. 2005, 127.8826 8828. (f) Kim, D. Y; Park, E. J. Org. Lett. 2002, 4, 545-547.

8. (a) Chambers, R. D.; Hutchinson, J. J. Fhorine Chem, 1998, 92, 45-52. (b) Chambers, R. D.; Greenhall, M. P.; Hutchinson, J. Tetrahedron 1996, 52, 1-8. (c) Resnati, G; DesMarteau, D. D. J. Org. Chem, 1991, 56, 4925-4929.

9. Frantz, R.; Hintermann, L.; Perseghini, M.; Broggini, D.; Togni, A. Org, Lett, 2003, 5, 1709-1712.

10. (a) Kang, Y. K.; Kim, D. Y. Tetrahedron Lett. 2006, 47, 4265-4568. (b) Cho, M. J.; Cho, M. G; Huh, S. C.; Kim, S. M.; Lee, K.; Koh, K. O.; Mang, J. Y. Bull. Korean Chem. Soc, 2006, 27, 857-861. (c) Park, E. J.; Kim, M. H.; Kim, D. Y. J. Org. Chem. 2004, 69, 6897. 6899. (d) Kim, D. Y.; Huh, S. C. Bull, Konean Chem. Soc, 2004 25, 347-348. (e) Kim, D. Y.; Kim, S. M.; Koh, K. O.; Mang, J. Y. Bull. Korean Chem. Soc. 2003, 24, 1425-1426. (f) Kim. D. Y.; Choi, Y. J.; Park, H. Y,; Joung, C. U.; Koh, K. O.; Mang, J. Y.; Jung, K.-Y. Sinth Conmint 2003, 33, 435-443. (g) Kim, D. Y, Huh, S. C.; Kim, S. M. Tetrahedron Lett. 2001, 42, 6299-630I. (h) Kim, D. Y.; Huh, S. C. Tetrahedron 2001, 57, 8933-8938.

11. Typical procedure for the fluorination of 1-chloro benzoylacetate 1a: To a stirred solution of I-chloro benzoylacetate 1a $(67.9 \mathrm{mg}, 0.3 \mathrm{mmol})$ and palladium catalyst $3 \mathrm{~d}(16.1 \mathrm{mg}, 0.015$ immol) in $\mathrm{MeOH}(1.5 \mathrm{~mL}$ ) was added NFSI (113.5 mg, 0.36 mmol) at room temperature. Reaction mixture was stirred for 118 $\mathrm{h}$ at room temperature. The mixture was diluted with saturated $\mathrm{NH}_{4} \mathrm{Cl}$ solution $(10 \mathrm{~mL})$ and extracted with ethyl acetate $(10 \mathrm{~mL} \times$ 3). The combined organic layers were dried over $\mathrm{MgSO}_{4}$, filtered, concentrated and purified by flash chromatography (ethyl acelate thexant $=1: 9)$ to afford 1 -chloro-l-fluoro benzoylacelate 4a $(66.1 \mathrm{mg} 90 \%):[\alpha]_{\mathrm{D}}^{21}=-18.1(c=0.695, \mathrm{CHCl}, 75 \%$ ee $) ;{ }^{\prime} \mathrm{H}$ NMR $\left(200 \mathrm{MHz}, \mathrm{CDCl}_{3}\right) 1.27(\mathrm{t}, J=7.1 \mathrm{~Hz}, 3 \mathrm{H}), 4.36$ (q. $J=7.2$ $\mathrm{Hz}, 2 \mathrm{H}), 7.50(\mathrm{~b}, J=7.8 \mathrm{~Hz}, 2 \mathrm{H}), 7.65(\mathrm{~L}, J=7.2 \mathrm{~Hz}, 1 \mathrm{H}), 8.06(\mathrm{~d}$ $J=7.8 \mathrm{~Hz}, 2 \mathrm{H}),{ }^{1} \mathrm{C}$ NMR $(50 \mathrm{MHz} . \mathrm{CDCl}) \delta[4.1,64.2,103.0$ (d, $J=263.1$ ), $128.8,130.9,130.1$ (d. $J=4$ ), $134.7,163.0$ (d, $J=$ $26.6), 184.6(\mathrm{~d}, J=26.8),{ }^{19} \mathrm{~F}$ NMR $\left(282 \mathrm{MHz}, \mathrm{CDCl}_{3}\right) \delta-117.05$, $\mathrm{R}_{\mathrm{t}} \mathrm{HPLC}(95.5$, nhexane: $: \mathrm{PrOH} .254 \mathrm{~mm}, 0.5 \mathrm{~mL} / \mathrm{min}$ ) Chiralcel $\mathrm{OB}-\mathrm{H}$ column, $\mathrm{l}_{\mathrm{R}}=12.7 \mathrm{~min}$ (minor), $\mathrm{l}_{\mathrm{R}}=13.7 \mathrm{~min}$ (major). 